\title{
Correlation between platelet count and outcome of chronic HCV patients treated with direct-acting antivirals
}

\section{Hosam M. Mohamed ${ }^{\text {a }}$, Shamardan E. S.Bazeed ${ }^{\text {b }}$, Heba A. Osman ${ }^{\text {b }}$, Hanan M.Fayed $^{\mathrm{c}}$}

${ }^{a}$ Qena Fever Hospital, Ministry of Health, Qena City, Egypt.

${ }^{\mathrm{b}}$ Department of Hepatology, Gastroenterology and Infectious diseases, Faculty of Medicine, South Valley University, Qena, Egypt.

${ }^{c}$ Department of Clinical and Chemical pathology, Faculty of Medicine, South Valley University, Qena, Egypt.

\begin{abstract}
:
Background: Egypt has the highest prevalence of chronic hepatitis $\mathrm{C}$ virus (HCV) infection. The direct acting anti-virus (DAAs) are available, with a reported 95\% sustained virological response after treatment for 12 weeks (12w-SVR).

Objectives: The current study aimed to assess the correlation between platelet count and the DAAs therapy outcome in chronic HCV (CHC) patients.

Patients and methods: three hundred CHC patients, Child-Pugh grade A, both naïve and treatment experienced patients were enrolled in this study, from outpatient Clinic, Department of Gastroenterology, Hepatology and Tropical medicine, Qena university hospital, Qena, Egypt, treated with DAAs for 12 weeks, either dual or triple therapy, according to criteria recommended by the national committee for chronic viral hepatitis (NCCVH). Patients categorized into three groups: (1) Group I (non-thrombocytopenic group): included $100 \mathrm{CHC}$ patients with platelet count $\geq 150\left(10^{9} / \mathrm{L}\right)$; (2) Group II (mild thrombocytopenic group): included $100 \mathrm{CHC}$ patients with platelet count 100-149 (10\%/L); (3) Group III (moderate thrombocytopenia): included $100 \mathrm{CHC}$ patients with platelet count $50-99\left(10^{9} / \mathrm{L}\right)$.

Results: The Overall CHC patient's mean age were (48.2 \pm 11$), 226(75.33 \%)$ were males and 74 (24.67\%) were females. 97.6\% (293/300)of CHC patients attainedSVR; $97 \%$ in (Group I), and 98 $\%$ in both (Group II), and (Group III), after 12 weeks DAAs therapy with no significant difference between groups.
\end{abstract}

Conclusion: both DAAs treatment modalities were efficient in the eradication of HCV; however, thrombocytopenia in CHC patients does not affect the DAAs therapy outcome.

Keywords: Direct-acting anti-virus, Platelets, SVR, HCV.

\section{Introduction:}

Hepatitis $\mathrm{C}$ virus (HCV) is an important etiology of chronic hepatitis and cirrhosisand is the leading indication for liver transplantation in adults around the World(Cavalcante and Lyra,2105).Global epidemiology of HCV infection

shows that the seroprevalence of Anti-HCV antibody has increased over the last decadefrom $2.3 \%$ to $2.8 \%$, corresponding to >

185 million infections

worldwide(MohdHanafiah et al., 2013).

In Egypt, $15 \%$ of an estimated population of 80 million is HCV positive, of which $93 \%$ are infected with genotype 4 (Ahmed et al., 2018).Before the advent of direct-acting antivirals (DAAs) therapy, the mainstay of $\mathrm{HCV}$ therapy involved interferon (IFN)-based regimens that had frequent contraindications, were poorly tolerated and achieved at best a $50 \%$ SVR rate(Yek et al., 2017). The introduction of DAAs has since revolutionized 
the HCV treatment landscape. SVR rates for DAA therapy exceed $90 \%$ in registration trials and are better tolerated than IFN-based regimens (Kohli et al., 2014). Understanding the probable outcome of treatment either before treatment (pre-treatment predictors) or during treatment (on-treatment predictors) is vital in determining the optimal therapy and duration of treatment. Several host genetic and viral factors are responsible for the variable outcome of patients with HCV infection (Navaneethan et al., 2009).

Thrombocytopenia in HCV patients occurs due to the interaction of many factors (Giannini, 2002) The challenge in the treatment of chronic HCV patients (CHC) with thrombocytopenia is the difficulty in initiating or maintaining therapy (Dienstag JL;McHutchisonJG,2006). The delay of therapy due to thrombocytopenia can result in diminished SVR and potential for progression of liver disease; additional medications may also be needed during their treatment (Afdhal et al., 2008).

\section{Aims of the Study:}

The aim of the current study was to assess the relation between platelet count and the directacting antivirals treatment outcome in $\mathrm{CHC}$ patients.

\section{Patients and methods:}

This was a prospective study involving 300 CHC Egyptian patients.

Setting: Outpatient Clinic, Department of Gastroenterology, Hepatology and Tropical medicine, Qena university hospital, Qena, Egypt.

The inclusion criteria were: age ranged between 18-70 years with HCV RNA positivity. The diagnosis of $\mathrm{CHC}$ was based on clinical and laboratory data, including (Positive anti HCV antibody and HCV PCR (more than $50 \mathrm{IU} / \mathrm{ml}$ ).

The exclusion criteria comprised patients with decompensated cirrhosis (Child grade B and
C) (Peng et al., 2016), Ascites, hepatic encephalopathy, pregnancy, hepatocellular carcinoma (HCC), HBV or HIV co-infection, renal impairment (GFR less than 30 $\mathrm{ml} /$ minute), those with INR $>1.7$, serum albumin $<2.8 \mathrm{~g} / \mathrm{dl}$, total bilirubin $>3 \mathrm{mg} / \mathrm{dl}$ or platelet count $<50\left(10^{9} / \mathrm{L}\right)$.

The study was directed according to the Declaration of Helsinki and its subsequent amendments, after approval by the institutional ethics committee review board, and obtaining informed consent from all patients. Patients were categorized into:

1) Group I (no thrombocytopenic group): included $100 \mathrm{CHC}$ patients with a platelet count $\geq 150\left(10^{9} / \mathrm{L}\right)$.

2) Group II (mild thrombocytopenic group): included $100 \mathrm{CHC}$ patients with a platelet count between 100- $149\left(10^{9} / \mathrm{L}\right)$.

3) Group III (moderate thrombocytopenia): included $100 \mathrm{CHC}$ patients with a platelet count between $50-99\left(10^{9} / \mathrm{L}\right)$.

All patients were submitted to clinical examination, laboratory testing, abdominal ultrasonography to assess hepatic echo pattern of the liver, the patency of portal vein, presence of splenomegaly and to exclude hepatocellular carcinoma.

All the patients were subjected to the following laboratory tests:

1) Complete blood count using cell dyne1800 (Abbot diagnostic Santa Clara-

California-USA)

2) Liver and kidney function assessment for serum alanine and aspartate aminotransferase (ALT, 0-41 U/L, and AST, 0-38 U/L, albumin, total bilirubin, urea $(10-50 \mathrm{mg} / \mathrm{dl})$, and creatinine (0.50-1.2 mg/dl); using Cobas c311 (Roche Diagnostic MannheimGermany). Prothrombin time (PT) using STA compact Max Coagulation system (StagoUSA)

3) Hepatitis markers for hepatitis B and hepatitis $\mathrm{C}$ were detected by an enzyme immunoassay (EIA) Cobas e411 (Roche Diagnostic Mannheim- Germany) 
4) HCV RNA viral load assessment by quantitative real-time polymerase chain reaction (QT-PCR) assay, using the CobasAmplicor, TaqMan HCV assay version 2.0 (Roche Diagnostic Mannheim, Germany) (the lower detection limit was $15 \mathrm{IU} / \mathrm{ml}$ ).

\section{Treatment regimen:}

Patients received DAAs therapy, according to the European Association for Study of Liver guidelines (EASL, 2015) Patients were monitored at Hepatology and infectious department, outpatient clinics Qena university hospital. 77 patients were treated for 12 weeks with once-daily dose combination therapy of Sofosbuvir (400mg) and Daclatasvir (60mg) and 223 patients were treated with triple combination therapy of Sofosbuvir, Daclatasvir, and Ribavirin.

\section{Monitoring of treatment efficacy:}

Virological assessment of HCV-RNA level was determined three times: at baseline (before treatment), at the end of treatment (week 12), and at 12 weeks post-treatment. Sustained virological response was considered when HCV RNA is less than the lower limit of detection (LLOD) at week 12 post-treatment (SVR12) by quantitative HCV-PCR while treatment failure was defined as confirmed HCV RNA above LLOQ 12 weeks posttreatment.

\section{Statistical analysis:}

Data were analyzed using a Statistical Program for Social Science (SPSS) version 18.0. Quantitative data were expressed as mean \pm standard deviation $(\mathrm{M} \pm \mathrm{SD})$. Qualitative data were expressed as frequency and percentage No(\%).Chi-square test:was used when comparing non-parametric data. A one-way analysis of variance (ANOVA), when comparing more than two means.Pvalues were considered statistically significant at $\mathrm{P}<0.05$.

\section{Results:}

Baseline characteristics:

A 300 CHC patients with the mean age of $(48.2 \pm 11$ years $)$ patients, treated for 12 weeks with DAAs either with two combination therapy $77(25.67 \%)$ or triple combination therapy 223(74.33\%). $243(81 \%)$ were treatment-naïve and $57(19 \%)$ treatment-experienced patients (Table 1). 226 of them (75.33\%) were male , $34(11.33 \%)$ were diabetic and $9(3 \%)$ were hypertensive. The mean BMI was 27.17 and the number of smokers was 223 patients (74.33\%). The mean HCV-RNA viral load level was $1.29 \times 10^{6}$.

According to ultrasonography findings, $50(16.6 \%)$ patients were cirrhotic, $134(44.6 \%)$ with abnormal echo pattern and $116(85.66 \%)$ were normal. Regarding laboratory profile, the mean of liver function tests, ALT , AST, AFP, bilirubin, platelet count, INR and albumin levels were $39.14 \mathrm{U} / \mathrm{L}, 34.27 \mathrm{U} / \mathrm{L}, 7.35 \mathrm{ng} / \mathrm{ml}, 0.81$ $\mathrm{mg} / \mathrm{ml}, 142.1\left(10^{9} / \mathrm{L}\right), 1.09$ and $3.98 \mathrm{~g} / \mathrm{ml}$ respectively (Table 1$)$.

The mean age of patients was 48.2, 52.5 and 44.1 years in group I, II and III respectively, with no significant difference between them. The number of males was $75 \%$ in group I, $76 \%$ in group II, $75 \%$ in group III with no significant difference between them. There was no significant difference between the studied groups as regard the association with other diseases DM, HTN, BMI and smoking (Table 2).

As regards the treatment, the number of naive patients was 77,72 and 94 in group I, II, III respectively (Table 2).

Regarding the pretreatment laboratory profile, results showed no significance difference between the studied groups as regard ALT, AST, Bilirubin, S.creatinine, fasting blood glucose, hemoglobin, WBCs and viral load. There was significant difference between the studied groups as 
regard serum albumin, INR and liver cirrhosis (Table 2).

Table (1): All CHC patients clinical and laboratory data

\begin{tabular}{|c|c|}
\hline Variables & All CHC patients \\
\hline $\begin{array}{ll}\text { Sex } & \\
0 & \text { Male } \\
& \text { Females } \\
\end{array}$ & $\begin{array}{l}\mathrm{N}(\%) \\
226(75.33 \%) \\
74(24.67 \%) \\
\end{array}$ \\
\hline Age (years) Mean \pm SD & $48.2 \pm 11$ \\
\hline $\begin{array}{ll}\text { Treatment regimen } \\
\text { Sofosbuvir\&Daclatasvir, } \\
\text { Sofosbuvir + Daclatasvir + Ribavirin }\end{array}$ & $\begin{array}{l}\mathrm{N}(\%) \\
77(25.67 \%) \\
223(74.33 \%) \\
\end{array}$ \\
\hline $\begin{array}{ll}\text { Past } & \text { treatment } \\
& \text { Naïve } \\
& \text { Experienced }\end{array}$ & $\begin{array}{l}\mathrm{N}(\%) \\
243(81 \%) \\
57(19 \%) \\
\end{array}$ \\
\hline BMI Mean \pm SD & $27.17 \pm 4.75$ \\
\hline $\begin{array}{ll}\text { Smoking } \\
\quad \text { Nonsmoker } \\
\quad \text { Smoker } \\
\end{array}$ & $\begin{array}{l}\mathrm{N}(\%) \\
223(74.33 \%) \\
77(25.67 \%) \\
\end{array}$ \\
\hline $\begin{array}{l}\text { Association with other chronic diseases } \\
\text { Non } \\
\text { DM } \\
\text { HTN }\end{array}$ & $\begin{array}{l}\mathrm{N}(\%) \\
257(85.66 \%) \\
34(11.33 \%) \\
9(3 \%)\end{array}$ \\
\hline ALT U/L (Mean \pm SD) & $39.14 \pm 29.7$ \\
\hline AST U/L (Mean \pm SD) & $34.27 \pm 21.36$ \\
\hline Alpha feto-protein $(\mathrm{ng} / \mathrm{ml})($ Mean \pm SD) & $7.35 \pm 17.12$ \\
\hline Bilirubin $\mathrm{mg} / \mathrm{dl}($ Mean $\pm \mathrm{SD})$ & $0.81 \pm 0.51$ \\
\hline Platelet count $\left(10^{9} / \mathrm{L}\right)($ Mean \pm SD $)$ & $142.1 \pm 65.87$ \\
\hline WBCs count $\left(10^{9} / \mathrm{L}\right)($ Mean \pm SD $)$ & $6020 \pm 1900$ \\
\hline Hemoglobin g/dl (Mean \pm SD) & $14.43 \pm 7.47$ \\
\hline Creatinine $\mathrm{mg} / \mathrm{dl}($ Mean $\pm \mathrm{SD})$ & $0.92 \pm 0.2$ \\
\hline Albumin g/dl (Mean \pm SD) & $3.98 \pm 0.5$ \\
\hline Fasting blood glucose $\mathrm{mg} / \mathrm{dl}(\mathrm{Mean}+\mathrm{SD})$ & $89.8 \pm 15.47$ \\
\hline INR $($ Mean \pm SD) & $1.09 \pm 0.11$ \\
\hline Viral load (million/ml) Mean \pm SD & $1.29 \pm 3.46$ \\
\hline $\begin{array}{l}\text { Ultrasonography findings } \\
\text { Normal liver } \\
\text { Abnormal echo pattern } \\
\text { Liver cirrhosis } \\
\end{array}$ & $\begin{array}{l}\mathrm{N}(\%) \\
116(85.66 \%) \\
134(44.66 \%) \\
50(16.66 \%) \\
\end{array}$ \\
\hline $\begin{array}{ll}\text { SVR } & \\
0 & \text { Responder } \\
& \text { Non-Responder }\end{array}$ & $\begin{array}{l}\mathrm{N}(\%) \\
293(97.66 \%) \\
7(2.33 \%)\end{array}$ \\
\hline
\end{tabular}


Table (2): clinical and laboratory data of three CHC studied groups

\begin{tabular}{|c|c|c|c|c|c|}
\hline & & Group I & Group II & Group III & $P$ value \\
\hline \multirow[t]{2}{*}{ Sex } & Male & $75(75 \%)$ & $76(76 \%)$ & $75(75 \%)$ & \multirow{2}{*}{0.982} \\
\hline & Female & $25(25 \%)$ & $24(24 \%)$ & $25(25 \%)$ & \\
\hline Age (years) & Mean \pm SD & $48.2 \pm 11.3$ & $52.6 \pm 7.8$ & $44.1 \pm 11.9$ & $<0.001$ \\
\hline \multirow[t]{2}{*}{ Treatment regimen } & SD & $77(77 \%)$ & $0(0 \%)$ & $0(0 \%)$ & \multirow{2}{*}{$<0.001$} \\
\hline & SDR & $23(23 \%)$ & $100(100 \%)$ & $100(100 \%)$ & \\
\hline \multirow[t]{2}{*}{ Past treatment } & naïve & 77 (77\%) & $72(72 \%)$ & $94(94 \%)$ & \multirow{2}{*}{$<0.001$} \\
\hline & Experienced & $23(23 \%)$ & $28(28 \%)$ & $6(6 \%)$ & \\
\hline BMI & Mean \pm SD & $27.3 \pm 4.5$ & $27.1 \pm 5.3$ & $27.2 \pm 4.6$ & 0.958 \\
\hline \multirow[t]{2}{*}{ Smoking } & Nonsmokers & $72(72 \%)$ & $75(75 \%)$ & $76(76 \%)$ & \multirow{2}{*}{0.797} \\
\hline & Smoker & $28(28 \%)$ & $25(25 \%)$ & $24(24 \%)$ & \\
\hline \multirow[t]{3}{*}{ Ch. Diseases } & Non & $82(82 \%)$ & $84(84 \%)$ & $91(91 \%)$ & \multirow{3}{*}{0.089} \\
\hline & DM & $12(12 \%)$ & $15(15 \%)$ & $7(7 \%)$ & \\
\hline & HTN & $6(6 \%)$ & $1(1 \%)$ & $2(2 \%)$ & \\
\hline $\begin{array}{l}\text { ALT U/L } \\
\text { (Mean } \pm \text { SD) }\end{array}$ & Mean \pm SD & $37.2 \pm 25.7$ & $38.3 \pm 30.5$ & $42.1 \pm 32.5$ & 0.469 \\
\hline $\begin{array}{l}\text { AST U/L } \\
(\text { Mean } \pm \text { SD })\end{array}$ & Mean \pm SD & $33.8 \pm 17.4$ & $33.9 \pm 23.9$ & $35.2 \pm 22.3$ & 0.883 \\
\hline $\operatorname{AFP}(\mathbf{n g} / \mathbf{m l})$ & Mean \pm SD & $5.3 \pm 7.7$ & $11.1 \pm 26.4$ & $5.6 \pm 10$ & 0.024 \\
\hline Bilirubin mg/dl & Mean \pm SD & $0.7 \pm 0.2$ & $0.9 \pm 0.4$ & $0.8 \pm 0.8$ & 0.031 \\
\hline PLT $\left(10^{9} / \mathbf{L}\right)$ & Mean \pm SD & $218.1 \pm 59.5$ & $129.6 \pm 13.5$ & $81.2 \pm 11.9$ & $<0.001$ \\
\hline $\mathrm{WBCs}\left(10^{9} / \mathrm{L}\right)$ & Mean \pm SD & $6.07 \pm 1.9$ & $5.7 \pm 2.1$ & $6.4 \pm 1.9$ & 0.027 \\
\hline Hemoglobin g/dl & Mean \pm SD & $15.4 \pm 12.6$ & $13.6 \pm 1.7$ & $14.3 \pm 1.5$ & 0.236 \\
\hline Creatinine mg/dl & Mean \pm SD & $0.9 \pm 0.2$ & $0.93 \pm 0.2$ & $0.9 \pm 0.2$ & 0.398 \\
\hline Albumin g/dl & Mean \pm SD & $4.1 \pm 0.4$ & $3.7 \pm 0.6$ & $4.1 \pm 0.4$ & $<0.001$ \\
\hline $\begin{array}{l}\text { Fasting blood } \\
\text { glucose mg/dl }\end{array}$ & Mean \pm SD & $90.7 \pm 16.2$ & $88.9 \pm 15.2$ & $88.1 \pm 18.8$ & 0.529 \\
\hline INR & Mean \pm SD & $1.06 \pm 0.06$ & $1.1 \pm 0.1$ & $1.08 \pm 0.07$ & $<0.001$ \\
\hline $\begin{array}{l}\text { Viral load } \\
\text { (million/ml) }\end{array}$ & Mean \pm SD & $1.02 \pm 1.7$ & $1.1 \pm 2.5$ & $1.7 \pm 5.2$ & 0.320 \\
\hline \multirow[t]{3}{*}{$\mathbf{U} / \mathbf{S}$} & Normal & $42(42 \%)$ & $28(28 \%)$ & $46(46 \%)$ & \multirow{3}{*}{0.0003} \\
\hline & DHP & $46(46 \%)$ & $42(42 \%)$ & $46(46 \%)$ & \\
\hline & L.C & $12(12 \%)$ & $30(30 \%)$ & $8(8 \%)$ & \\
\hline
\end{tabular}


Table (3):Comparison between the three studied groups as regard SVR

\begin{tabular}{|l|l|l|l|l|l|}
\hline \multicolumn{2}{|l|}{ Variables } & Group I & Group II & Group III & P-value \\
\hline \multirow{2}{*}{ SVR } & Responder & $97(97 \%)$ & $98(98 \%)$ & $98(98 \%)$ & \multirow{2}{*}{0.8639} \\
\cline { 2 - 5 } & Non responder & $3(3 \%)$ & $2(2 \%)$ & $2(2 \%)$ & \\
\hline
\end{tabular}

This table shows no statistical significant difference $(\mathbf{p}$-value $>\mathbf{0 . 0 5})$ between the studied three groups as regard SVR.

\section{Treatment efficacy:}

Our study results show that the overall patient's SVR was $97.6 \%$ (293/300). The rate of SVR was $97 \%$ in patients with normal platelet count (Group I), and $98 \%$ among those with mild thrombocytopenia
(Group II), and $98 \%$ among those with moderate thrombocytopenia (Group III), with no significant difference between these groups.

Table (4): Pretreatment Demographic Data in Responders and Non responders:-

\begin{tabular}{|c|c|c|c|}
\hline Parameter & $\begin{array}{c}\text { Responders } \\
(\mathbf{n}=\mathbf{2 9 3})\end{array}$ & $\begin{array}{c}\text { Non Responders } \\
(\mathbf{n}=7)\end{array}$ & P-value \\
\hline Age (years) Mean \pm SD & $48.24 \pm 10.9$ & $47.57 \pm 13.07$ & 0.8737 \\
\hline $\begin{array}{ll}\text { Sex } & \\
\text { Male } & \mathrm{n}(\%) \\
\text { Females } & \mathrm{n}(\%) \\
\end{array}$ & $\begin{array}{l}220(57.1 \%) \\
73(24.9 \%)\end{array}$ & $\begin{array}{l}6(85.7 \%) \\
1(14.3 \%)\end{array}$ & 0.6695 \\
\hline \begin{tabular}{cc}
\multicolumn{2}{l}{ Treatment regimen } \\
SD & $\mathbf{n}(\%)$ \\
SDR & $\mathbf{n}(\%)$ \\
\end{tabular} & $\begin{array}{c}74(25.2 \%) \\
219(74.8 \%) \\
\end{array}$ & $\begin{array}{l}3(42.85 \%) \\
4(57.15 \%) \\
\end{array}$ & 0.2920 \\
\hline $\begin{array}{l}\text { Past treatment } \\
\text { Naïve n }(\%) \\
\text { Experienced } \mathbf{n}(\%)\end{array}$ & $\begin{array}{l}236(80.54 \%) \\
57(19.46 \%)\end{array}$ & $\begin{array}{c}7(100 \%) \\
0(0 \%)\end{array}$ & 0.7322 \\
\hline BMI kg/m2 Mean \pm SD & $27.17 \pm 4.78$ & $27.17 \pm 3.5$ & 0.9983 \\
\hline $\begin{array}{ll}\text { Smoking } & \\
\text { Non smoker } & \text { n }(\%) \\
\text { Smoker } & \text { n }(\%)\end{array}$ & $\begin{array}{l}218(25.6 \%) \\
75(74.4 \%)\end{array}$ & $\begin{array}{l}5(71 \%) \\
2(29 \%)\end{array}$ & 0.8587 \\
\hline $\begin{array}{l}\text { Association with other } \\
\text { chronic diseases } \\
\text { DM n (\%) } \\
\text { HTN n (\%) } \\
\end{array}$ & $\begin{array}{l}34(11.6 \%) \\
8(0.27 \%)\end{array}$ & $\begin{array}{c}0(0 \%) \\
1(14.3 \%) \\
\end{array}$ & 0.545 \\
\hline ALT U/L $($ Mean \pm SD $)$ & $39.17 \pm 29.93$ & $39.71 \pm 18.74$ & 0.481 \\
\hline AST U/L (Mean \pm SD) & $34.18 \pm 21.42$ & $37.14 \pm 18.23$ & 0.718 \\
\hline $\operatorname{Afp}(\mathrm{ng} / \mathrm{ml})($ Mean \pm SD $)$ & $7.38 \pm 17.32$ & $6.71 \pm 2.6$ & 0.920 \\
\hline Bilirubin mg/dl (Mean \pm SD) & $0.79 \pm 0.29$ & $1.9 \pm 2.5$ & $<0.00001$ \\
\hline Platelets $\left(10^{9} / \mathrm{L}\right)($ Mean \pm SD $)$ & $142.59 \pm 66.39$ & $127.29 \pm 33.15$ & 0.545 \\
\hline WBCs $\left(10^{9} / \mathrm{L}\right)($ Mean \pm SD $)$ & $6.06 \pm 1.99$ & $4.58 \pm 1.4$ & 0.0527 \\
\hline Hb g/dl (Mean \pm SD) & $14.46 \pm 7.52$ & $13.16 \pm 1.3$ & 0.654 \\
\hline $\begin{array}{l}\text { Createnine mg/dl } \\
(M e a n \pm S D)\end{array}$ & $0.92 \pm 0.20$ & $0.83 \pm 0.13$ & 0.2313 \\
\hline Albumin g/dl (Mean \pm SD) & $3.98 \pm 0.52$ & $4.1 \pm 0.35$ & 0.533 \\
\hline FBG mg/dl (Mean \pm SD) & $89.58 \pm 16.24$ & $88 \pm 12$ & 0.7996 \\
\hline
\end{tabular}




\begin{tabular}{|c|c|c|c|}
\hline INR $($ Mean \pm SD $)$ & $1.09 \pm 0.11$ & $1.07 \pm 0.05$ & 0.7187 \\
\hline $\begin{array}{c}\text { Viral load }(\text { million} / m l) \\
\text { Mean } \pm \text { SD }\end{array}$ & $1.29 \pm 3.49$ & $1.35 \pm 1.96$ & 0.963 \\
\hline $\begin{array}{l}\text { Ultrasonography findings } \\
\text { Normal liver } \mathrm{n}(\%) \\
\text { Abnormal echopattern } \mathrm{n} \\
(\%) \\
\text { Liver cirrhosis } \mathrm{n}(\%)\end{array}$ & $\begin{array}{c}114(38.9 \%) \\
134(45.73 \%) \\
45(15.37 \%)\end{array}$ & $\begin{array}{c}2(29 \%) \\
0(0 \%) \\
5(71 \%)\end{array}$ & 0.002 \\
\hline
\end{tabular}

\section{Discussion:}

Chronic hepatitis $\mathrm{C}$ infection is a major cause of chronic liver disease, cirrhosis and liver cancer(Hessel et al., 2016). The introduction of the direct acting antiviral agents (DAAs) in the treatment of $\mathrm{HCV}$ infections has revolutionized the field. DAAs therapy now means that the majority of patients can be successfully cured with ever decreasing durations of well tolerated regimens (Hathorn;Elsharkawy AM, 2016).

The findings of the current study showed that the sustained virological response (SVR) was $97.6 \% \quad(293 / 300)$ Overall patients. Rates of sustained virological response were $97 \%$ in patients with no thrombocytopenia (Group I), and $98 \%$ among those with mild thrombocytopenia (Group II), and $98 \%$ among those with moderate thrombocytopenia (Group III), with no significant difference between these groups. Our study did not find a statistically significant association between sustained virological response and platelet count.

The results of the current study were supported by Babatin et al.,(2017)., A reallife prospective observational study in which 40 patients received the combination of SOF $400 \mathrm{mg}$ daily and DCV 60mg daily. All patients were native Saudis with HCV-GT4 infection, and all completed 12 weeks of treatment. The mean age was $49.5 \pm 14.5$ years and $62.5 \%$ were men.

Twenty-onepatients (52.5\%) had failed prior IFN-based treatment-Cirrhosis was present in $35.9 \%$ All patients achieved SVR12.

Regarding the effect of Low platelet count on the SVR, The present study showed no significant correlation between thrombocytopenia and Virological Failure. These results agreed with Zaghloul et al., (2015).A prospective cohort study included 260 chronic HCV patients stratified into 2 groups; group I included 60 patients with platelet count $\geq 150,000 / \mathrm{mm} 3$ as a disease control and group II (thrombocytopenia group) included 200 patients with platelet count ranged from 30,000- 149,000/mm3. Patients were subjected to dual or triple therapy, according to criteria recommended by the national committee for chronic viral hepatitis (NCCVH) 2014. Sustained virological response and relapse in patients with thrombocytopenia didn't significantly differ from normal platelets patients. These results disagreed with Elsharkawy et al.,(2017),which reported that low platelet count was significantly associated with treatment failure as this factor might be associated with more advanced liver fibrosis. 


\section{Conclusion:}

Both DAAs treatment modalities were efficient in the eradication of $\mathrm{HCV}$; however, thrombocytopenia in $\mathrm{CHC}$ patients does not affect the DAAs therapy outcome.

\section{Conflict of Interest:}

The authors have no conflict of interest related to this publication.

\section{References:}

Afdhal N, McHutchison J, Brown R, Brown R, Jacobson I, Manns M, Poordad F et al., (2008).Thrombocytopenia associated with chronic liver disease. J Hepatol, 48 (6):10001007.

\section{Ahmed OA,Safwat E, Khalifa MO, Elshafie AI1, Fouad MHA, Salama MM et al., (2018).Sofosbuvir Plus Daclatasvir in Treatment of Chronic Hepatitis C Genotype 4 Infection in a Cohort of Egyptian Patients:An Experiment the Size of Egyptian Village. International Journal of Hepatology, Vol. 2018:1-5.}

Babatin M. A, Alghamdi S. A, Albenmousa A, Alaseeri A, Aljarodi M, Albiladi H et al., (2017). Efficacy and Safety of Simeprevir or Daclatasvir in Combination WithSofosbuvir for the Treatment of Hepatitis C Genotype 4 Infection. J ClinGastroenterol, 52 (5):452457.

Cavalcante LN,Lyra AC. (2015). Predictive factors associated with hepatitis C antiviral therapy response. World J Hepatol,7(12): 1617-1631.

Dienstag JL,McHutchisonJG. (2006). American Gastroenterological Association medical position statement on the management of hepatitis $\mathrm{C}$ Gastroenterology, 130(1):225-230.

European Association for Study of Liver,(2015).EASL Recommendations on Treatment of Hepatitis C 2015. J Hepatology, 63(1):199-236.

Giannini EG.(2002).Thrombocytopenia in chronic liver disease and pharmacologic treatment options. Aliment PharmacolTher,23(8):1055-1065.

Hathorn E,ElsharkawyAM. (2016).Management of hepatitis $C$ genotype 4 in the directly acting antivirals era BMJ Open Gastro, 3(1):e000112.

Hessel MH, Cohen AF, Rissmann R. (2016).Sofosbuvir and Daclatasvir . $\mathrm{Br} \mathrm{J}$ ClinPharmacol , 82(3): 878-879.

Kohli A, Shaffer A, Sherman A, Kottilil S. (2014). Treatment of hepatitis C:a systematic review. JAMA, 312(6):631-640.

Elsharkawy A, Fouad R, EL AkelW, El Raziky M, Hassany M, Shiha G et al., (2017).Sofosbuvir-based treatment regimens :real life results of 14409 chronic HCV genotype 4patientsinEgypt.Aliment. PharmacolTher, 45(5):681-687.

MohdHanafiah K, Groeger J, Flaxman AD, Wiersma ST.(2013).Global epidemiology of hepatitis $\mathrm{C}$ virus infection: new estimates of age-specific antibody to HCV seroprevalence. Hepatology, 57(4):1333-42.

Navaneethan U, Kemmer N, Neff G. (2009).Predicting the probable outcome of treatment in $\mathrm{HCV}$ patients. TherAdvGastroenterol, 2(5):287302.

Peng Y, QI X, Guo X. (2016). Child-Pugh versus MELD score for the assessment of 
prognosis in liver cirrhosis (2016). A systematic review and META - Analysis of observational studies, Medicine (Baltimore),95(8):e2877.

Yek C, De la Flor C, Marshall J, Zoellner C, Thompson G, Quirk $\mathrm{L}$ et al., (2017).Effectiveness of direct-acting antiviral therapy for hepatitis $\mathrm{C}$ in difficultto-treat patients in a safety-net health system: a retrospective cohort studyBMC Medicine,15(1):204.

Zaghloul S. G, Hammam A. A, Wadea F. M, Saeed M. R. (2015): Outcome ofSofosbuvir Containing Treatment Regimens in Egyption Chronic Hepatitis C patients with Thrombocytopenia. International Journal of Science and Research, 6 (1): 321-327. 